Received: 13.09 .2018

Revised: 18.01.2019

Accepted: 15.02 .2019

DOI: $10.17804 / 2410-9908.2019 .1 .043-054$

\title{
EXPERIENCE OF APPLYING SHOT-TERM INTERMEDIATE ANNEALS IN BACK-PRESSURE EQUAL-CHANNEL ANGULAR PRESSING OF ALUMINUM ALLOYS
}

\author{
V. A. Andreyachshenko* \\ Ekibastuz Technical and Engineering Institute Named After Academician K. Satpayev, \\ 54 A Energetikov St. Ekibastuz, 141200, Kazakhstan \\ (iD https://orcid.org/0000-0001-6933-8163 ख Vi-ta.z@mail.ru \\ *Corresponding author. E-mail: Vi-ta.z@ mail.ru \\ Address for correspondence: 54 "A” Energetikov St., 141200, Ekibastuz, Kazakhstan \\ Tel.: +7 (701) 3962994
}

The influence of severe plastic deformation on hypoeutectic silumin is studied. A positive effect of equal-channel angular pressing with back pressure on the mechanical properties of the AL9 alloy of the Al-Si-Mn-Fe system has been found experimentally. The evolution of the microstructure in the process of deformation is estimated. The effect of short-term intermediate anneals on the microstructure, mechanical properties and ultimate plasticity of the alloy is studied. As a result of additional annealing, significant microstructural changes do not succeed, but the coagulation of excess phases proceeds very intensively, which causes a $20 \%$ increase in strength after the second deformation cycle. Fractographic studies demonstrate the presence of coagulated particles on the samples deformed with the use of short-term intermediate anneals. Limiting plasticity also rises to 0.2 as compared to 0.12 for the samples treated with only ECAP-BP.

Keywords: hypoeutectic silumins, AL9, Al-Si-Mn-Fe, equal-channel angular pressing with back pressure.

\section{References}

1. Senatorova O.G., Grushko O.E., Tkachenko E.A., Antipov V.V., Molostova I.I., Sidel'nikov V.V., Legoshina S.F. New high-strength aluminum alloys and materials. Tekhnol. Legk. Splavov, 2007, no. 2, pp. 17-24. (In Russian).

2. Mironov A.E. Kotova E.G. Development of new marks of welded aluminum antifriction alloys for exchange the bronze in joints of friction. Izv. Samarsk. Nauch. Tsentra Ross. Akad. Nauk, 2011, vol. 13, pp. 1136-1140. (In Russian).

3. Klochkova Yu.Yu., Grushko O.E., Lantsova L.P., Burlyaeva I.P., Ovsyannikov B.V. The assimilation for industrial production of semi-finished products from advanced aluminumlithium alloy V-1469. Aviats. Mater. Technol., 2011, no. 1, pp. 8-12. (In Russian).

4. Docenko Yu.V., Seliverstov V.Yu. Features of solidification casting of aluminum alloys with increasing pressure and modification. Eastern-European Journal of Enterprise Technologies, 2012, no. 1 (5), pp. 18-22. (In Russian).

5. Selivanov A.A., Antipov K.V., Astashkin A.I., Ovsyannikov B.V. Optimization of artificial aging of forged and rolled semi-finished products from heat-resistant aluminum alloy AK4-1h. Trudy VIAM, 2018, no. 4 (64), pp. 9-19. DOI: 10.18577/2307-6046-2018-0-4-9-19. (In Russian).

6. Marukovich E.I., Stetsenko V.Y., Gutev A.P. Manufacture and use of silumin with globular silicon. Litiyo i Metallurgiya (Foundry Production and Metallurgy), 2017, no. 2 (87), pp. 15-19. DOI: 10.21122/1683-6065-2017-2-15-19. (In Russian). 
7. Prudnikov A.N., Popova M.V., Prudnikov V.A. Effect of deformation on the structure and properties of silumin. Bulletin of the Siberian State Industrial University, 2017, no. 3 (21), pp. 11-17. (In Russian).

8. Bogatov A.A., Nukhov D.Sh., Leshev I.V. Development of innovative processes metal forming through intensive alternating strain. Processing of Solid and Laminate Materials, 2016, no. 1 (44), pp. 35-43. (In Russian).

9. Spuskanyuk V.Z., Berezina A.L., Dubodelov V.I., Davydenko O. A., Fixssen V. N., Sliva K.I., Monastyrska T.O., Gangalo A.N. Microstructure and mechanical properties of the hypereutectic Al-Si alloy after several plastic deformation. Metallofiz. Noveishie Tekhnol., 2014, vol. 36, no. 5, pp. 649-660. DOI: 10.15407/mfint.36.05.0649. (In Russian).

10. Bochvar N.R., Rohlin L.L., Tarytina I.E. Combined effect of sever plastic deformation and cold rolling on mechanical properties of the Al-Mg2Si system alloys. Perspektivnye Materialy, 2018, no. 7, pp. 49-56. DOI: 10.30791/1028-978x-2018-7-49-56. (In Russian).

11. Avtokratova E.V., Sitdikov O.Sh., Markushev M.V. Superplasticity of Al-Mg-Sc(Zr) alloys, subjected to intense plastic deformation. Materials Physics and Mechanics, 2017, vol. 33, no. 1, pp. 19-28. DOI: 10.18720/MPM.3312017_3. (In Russian).

12. Polishchuk S.S., Berezina A.L., Davidenko A.A., Spuskanyuk V.Z., Fixsen V.N., Yashchenko A.V., Burkhovetskii V.V. Structure and Properties of A356-AlCuFe Composite Obtained Using Electromagnetic Agitation. Metallofiz. Noveishie Tekhnol., 2014, vol. 36, no. 9, pp. 1189-1205. DOI: 10.15407/mfint.36.09.1189. (In Russian).

13. Brodova I.G., Petrova A.N., Razorenov S.V., Shorohov E.V. Resistance of submicrocrystalline aluminum alloys to high-rate deformation and fracture after dynamic channel angular pressing. The Physics of Metals and Metallography, 2015, vol. 116, iss. 5, pp. 519-526. DOI: 10.1134/S0031918X15050051.

14. Kocich R., Fiala J., Szurman I., Macháčková A., Mihola M. Twist-channel angular pressing: effect of the strain path on grain refinement and mechanical properties of copper. Journal of Materials Science, 2011, vol. 46, no. 24, pp. 7865-7876. DOI: 10.1007/s10853-011-5768-1.

15. Markushev M.V., Sloboda V.N., Kaibyshev O.A. Method for deformation working of materials and apparatus for performing the same. RF Patent 2146571, 2000. (In Russian).

16. Mckenzie P.W.J., Lapovok R. ECAP with back pressure for optimum strength and ductility in aluminium alloy 6016. Part 2: Mechanical properties and texture. Acta Materialia, 2010, vol. 58 (9), pp. 3212-3222. DOI: 10.1016/j.actamat.2010.01.037.

17. Medvedev Alexander E., Lapovok Rimma, Koch Eric, Höppel Heinz Werner, Göken Mathias. Optimisation of interface formation by shear inclination: Example of aluminiumcopper hybrid produced by ECAP with back-pressure. Materials \& Design, 2018, vol. 146, pp. 142-151. DOI: 10.1016/j.matdes.2018.03.021.

18. Naizabekov A.B., Andreyashchenko V.A. Evaluation of possibility for increase of mechanical characteristics of the $\mathrm{Al}-\mathrm{Fe}-\mathrm{Si}-\mathrm{Mn}$ alloy by equal-channel angular pressing. Metallurgist, 2013, vol. 57, no. 1-2, pp. 159-163. DOI: 10.1007/s11015-013-9706-0.

19. Cepeda-Jiménez C.M., García-Infanta J.M., Zhilyaev A.P., Ruano O.A., Carreno F. Influence of the supersaturated silicon solid solution concentration on the effectiveness of severe plastic deformation processing in Al-7wt.\% Si casting alloy. Materials Science and Engineering: A, 2011, vol. 528, pp 7938-7947. DOI: 10.1016/j.msea.2011.07.016.

20. Mochalov N.A., Galkin A.M., Mochalov S.I., Parfenov D.Yu. Plastometric Studies of Metals. Moscow, Intermet Engineering Publ., 2003, 318 p. (In Russian).

21. Cepeda-Jimenez C.M., Orozco-Caballero A., Garcia-Infanta J.M., Zhilyaev A.P., Ruano O.A., Carreno F. Assessment of homogeneity of the shear-strain pattern in Al-7wt\%Si casting alloy processed by high-pressure torsion. Materials Science and Engineering A, 2014, vol. 597, pp. 102-110. DOI: $10.1016 /$ j.msea.2013.12.072. 
22. Andreyachshenko V., Naizabekov A. The technology of equal channel angle backpressure extrusion for deformation iron and aluminium alloys. In: Proceedings Book of 3rd International Conference NANOCON, Czech Republic, Tanger Ltd., 2011, pp. 246-252.

23. Naizabekov A., Andreyachshenko V., Kliber J. Forming of microstructure of the Al-Si-Fe-Mn system alloy by equal channel angular pressing with backpressure. In: Proceedings of 21st International Conference on Metallurgy and Materials Metal-2012, Brno Czech Republic, 2012, pp. 391-395.

24. Naizabekov A., Andreyachshenko V. Microstructural and mechanical characteristics of the Al-Fe-Si-Mn alloy subjected to equal-channel angular pressing. In: Sbornik trudov X Mezhdunarodnoy konferentsii «Perspektivnye tekhnologii, oborudovanie i analiticheskie sistemy dlya materialovedeniya $i$ nanomaterialov» [Transactions of the $10^{\text {th }}$ International Conference on Advanced Technologies, Equipment and analytical Systems for Materials Science and Nanomaterials]. Almaty, Kazakhstan, 2013, pp. 114-122. (In Russian). 
Подана в журнал: 13.09 .2018

УДК 621.771

DOI: $10.17804 / 2410-9908.2019 .1 .043-054$

\title{
ОПЫТ ПРИМЕНЕНИЯ КРАТКОСРОЧНЫХ ПРОМЕЖУТОЧНЫХ ОТЖИГОВ ПРИ РАВНОКАНАЛЬНОМ УГЛОВОМ ПРЕССОВАНИИ С ПРОТИВОДАВЛЕНИЕМ АЛЮМИНИЕВЫХ СПЛАВОВ
}

\author{
В. А. Андреященко \\ Екибастузский инженерно-технический институт им. академика К. Сатпаева, \\ г. Экибастуз, Казахстан \\ a) (iD https://orcid.org/0000-0001-6933-8163 Vi-ta.z@ mail.ru \\ Ответственный автор. Электронная почта: Vi-ta.z@mail.ru \\ Адрес для переписки: 141208, г. Экибастуз, ул. Энергетиков 54 «А», Республика Казахстан \\ Тел.: +7 (701) 396-29-94
}

Изучено влияние интенсивной пластической деформации на доэвтектические силумины. Опытным путем установлено положительное влияние равноканального углового прессования с противодавлением на механические свойства сплава системы Al-Si-Mn-Fe типа AЛ9. Оценена эволюция микроструктуры в процессе деформирования. Изучен эффект применения краткосрочных промежуточных отжигов на микроструктуру, механические свойства и предельную пластичность сплава. В результате проведения дополнительных отжигов существенные микроструктурные изменения не успевают реализоваться, однако процесс коагуляции избыточных фаз протекает весьма интенсивно, что вызывает рост прочности в целом на 20 \% после второго цикла. Фрактографические исследования демонстрируют присутствие коагулированных частиц на образцах, деформируемых с использованием краткосрочных промежуточных отжигов. Предельная пластичность также повышается до 0,2 в сравнении с образцами, обработанными только РКУПП с противодавлением - 0,12.

Ключевые слова: доэвтектические силумины, АЛ9, Al-Si-Mn-Fe, равноканальное угловое прессование с противодавлением.

\section{1. Введение}

В технике алюминиевые сплавы находят все большее и большее распространение. Отчасти это связано с разработкой новых сплавов [1-3], применением модифицирования [4], отработкой режимов производства $[5,6]$ и т. д. Повышенный интерес к алюминиевым сплавам вызван прежде всего их уникальными свойствами, сочетающими прочность, пластичность, коррозионную стойкость, герметичность, жидкотекучесть, стойкость в различных кислотах и, особенно, малый вес.

Именно за счет этих характеристик алюминиевые сплавы успешно применяются для производства деталей и узлов в космической технике, машиностроении и др. Особенно популярны литейные алюминиевые сплавы типа АЛ9, применяемые для изготовления разнообразных литых деталей машин. Ряд деталей, таких как поршни и др., можно изготавливать как методами литья, так и деформационными методами. В последнем случае литейные алюминиевые сплавы малопригодны. Однако существует опыт повышения комплекса физикомеханических характеристик литейных сплавов методами термомеханической обработки [7]. Кроме того, в качестве упрочняющих деформационных методов хорошо зарекомендовали себя методы, реализующие интенсивные пластические деформации [8-11], одним из которых является равноканальное угловое прессование [12]. При этом помимо классических методов РКУПП также известны их вариации [13], например, Тwist-канальное угловое прессование [14], РКУПП 
с сужающимся или расширяющимся каналом [15], РКУПП с противодавлением [16], обеспечиваемым вторым пуансоном, движущимся противоположно течению металла [17], РКУПП со ступенью в выходном канале инструмента [18]. Варианты реализации РКУПП направлены, как правило, на устранение растягивающих напряжений, которые образуются в области внутреннего угла при переходе из входного канала в выходной, и на устранение эффекта не полного заполнения выходного канала инструмента, что в свою очередь связано с частичным переходом периферийных слоев материала заготовки с торцевой поверхности на боковую, что вызывает также искажение формы переднего торца заготовки и повышение объема обрези. Эффективность РКУПП для обработки доэвтектических силуминов уже подтверждена [19]. Ранее уже был изучен положительный опыт улучшения литейных алюминиевых сплавов типа АЛ9 методом равноканального углового прессования со ступенью в выходном канале инструмента. Причем обработка велась как в холодном, так и в горячем состояниях. Также выполнен анализ влияния этих обработок на предельную пластичность сплава.

Цель настоящей работы - исследование влияния краткосрочных промежуточных отжигов при реализации РКУПП для повышения ресурса доэвтектического алюминиевого сплава типа АЛ9.

\section{2. Материалы и методы исследования}

В качестве материала исследования выбран литейный алюминиевый сплав типа АЛ9 со следующим составом: $4,0 \% \mathrm{Si}-1,12 \% \mathrm{Mn}-0,5 \% \mathrm{Fe}-0,32 \% \mathrm{Cu}$, остальное Al. Bce образцы были предварительно отожжены. В дальнейшем для измельчения микроструктуры и улучшения свойств сплава проведено РКУПП в инструменте со ступенью в выходном канале при комнатной температуре. Проведение операции отжига имело две цели: 1) растворение хрупких интерметаллических составляющих и 2) сфероидизация избыточных фаз. Была изучена микроструктура сплава, определены механические свойства при испытании на разрыв. Микроструктура до и после обработки исследована на поперечных микрошлифах на оптическом микроскопе Leica и на просвечивающем электронном микроскопе JEM2100, микрофратографические исследования выполнены на сканирующем электронном микроскопе JSM5910. Механические испытания проводились на крутильно-разрывной машине МИ-40КУ. Первичная диаграмма нагрузка-перемещение переведена в координаты напряжение-деформация. Напряжение определено отношением нагрузки к начальной площади поперечного сечения образцов. Деформации вычислены при помощи соотношения: $\varepsilon=\Delta l / l_{0} \cdot 100 \%$. Пластичность в области однородной деформации (до образования шейки) определена из выражения [20] $\Lambda=1,73 \ln [100 /(100-\delta)]$, где $\delta$ - относительное удлинение; предельная пластичность в области неоднородной деформации из выражения: $\Lambda \mathrm{p}=1,73 \ln [100 /(100-\psi)]$, где $\psi$ - относительное сужение. Суммарная степень деформации при РКУПП определена по методике, приведенной в работе [18].

\section{3. Обсуждение результатов}

Характерной преддеформационной обработкой доэвтектических силуминов является отжиг с медленным охлаждением в печи. Отожженные образцы сплава имеют высокое содержание коагулированных кремниевых выделений размером до 10 мкм и выделения избыточных фаз размером менее 1 мкм. При исследовании на оптическом микроскопе выделения размером менее 1 мкм плохо выявляются. Наиболее отчетливо такая микроструктура наблюдается на фотографии, полученной при помощи просвечивающей электронной микроскопии (рис. 1). Кремниевые частицы имеют форму неправильных призм со скругленными очертаниями. Интерметаллидные фазы представлены преимущественно игольчатыми железосодержащими частицами и сферическими включениями, содержащими медь и марганец. Высокая концентрация включений не позволяет выявить границы зерен $\alpha$-твердого 
раствора основной матрицы. В общем, данные результаты согласуются с ранее проведенными исследованиями [21].

Отожженные образцы далее были подвергнуты РКУПП при комнатной температуре с количеством циклов деформирования от 1 до 3. Проведение большего количества циклов затруднительно в связи с образованием поперечных трещин и разрывов на образцах. Такое деформационное поведение вызвано прежде всего спецификой микроструктуры. Образцы, имеющие деформационные дефекты, выявляемые невооруженным взглядом, к дальнейшему деформированию не допускаются. На рис. 2 представлено изображение микроструктуры, полученное при помощи оптической микроскопии. На всех образцах наблюдается высокая дисперсность структуры. Увеличение количества циклов деформирования приводит к образованию «квазиэвтектического» рисунка, причем на микрошлифах, полученных без применения промежуточных отжигов, этот эффект заметен более отчетливо.

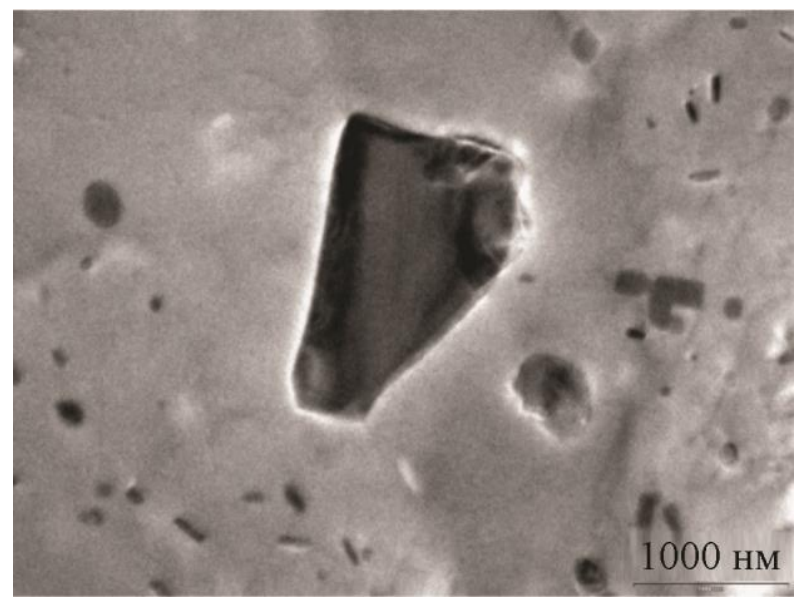

Рис. 1. Микроструктура отожженного образца, ПЭМ

С увеличением количества циклов прочностные характеристики сплава имеют тенденцию к увеличению, однако наблюдается снижение характеристик пластичности (рис. 3). Такая тенденция характерна для материалов, обработанных методами интенсивной пластической деформации [22], в том числе доэвтектических сплавов системы Al-Si-Mn-Fe [23].

$\mathrm{C}$ целью повышения ресурса сплава решено использовать кратковременнные промежуточные отжиги при температуре $200{ }^{\circ} \mathrm{C}$ в течение 5 мин после каждого цикла. Первый промежуточный отжиг осуществлен между первым и вторым циклами деформирования, таким образом, отличия заметны уже после двух циклов деформирования. Использование промежуточных отжигов позволило повысить предел прочности после двух циклов РКУПП до $455 \mathrm{MПа,} \mathrm{что} \mathrm{превышает} \mathrm{режим} \mathrm{без} \mathrm{отжига} \mathrm{более} \mathrm{чем} \mathrm{на} 20 \%$. Однако это вызвало резкое сокращение относительного удлинения (на $90 \%$ ) до величины $11 \%$. Хотя для рассматриваемого сплава это значение является вполне приемлемым. Как показывают изображения микроструктуры, приведенные на рис. 2, в результате проведения отжигов изменений микроструктуры не происходит. Несмотря на то, что температура соответствует температуре старения, в течение 5 мин интенсивного распада твердого раствора не происходит. Учитывая, что в качестве предварительной обработки использован отжиг, твердый раствор не является пересыщенным, а кратковременный отжиг способствует коагуляции интерметаллидных фаз и частиц кремния. 


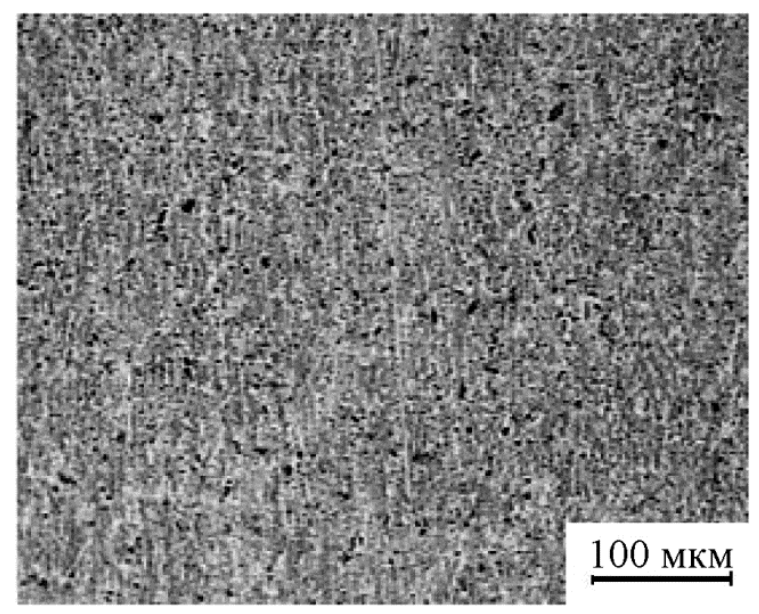

$a$

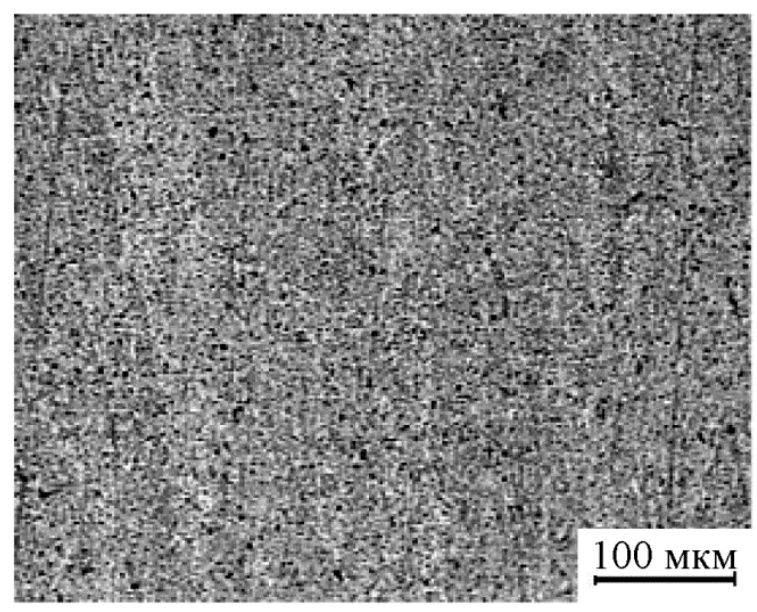

B

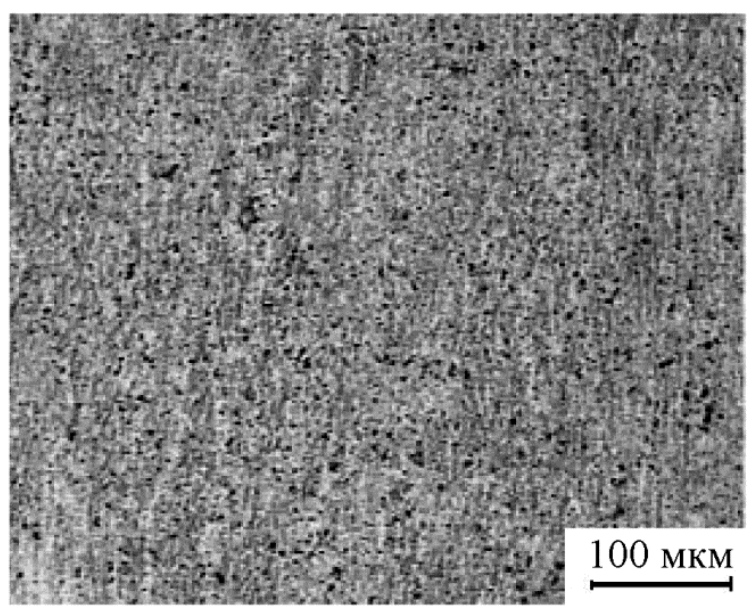

$\sigma$

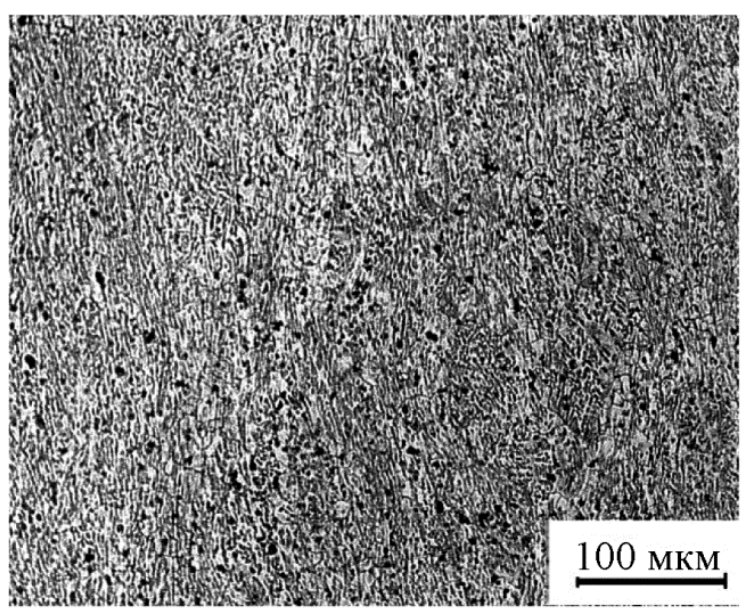

2

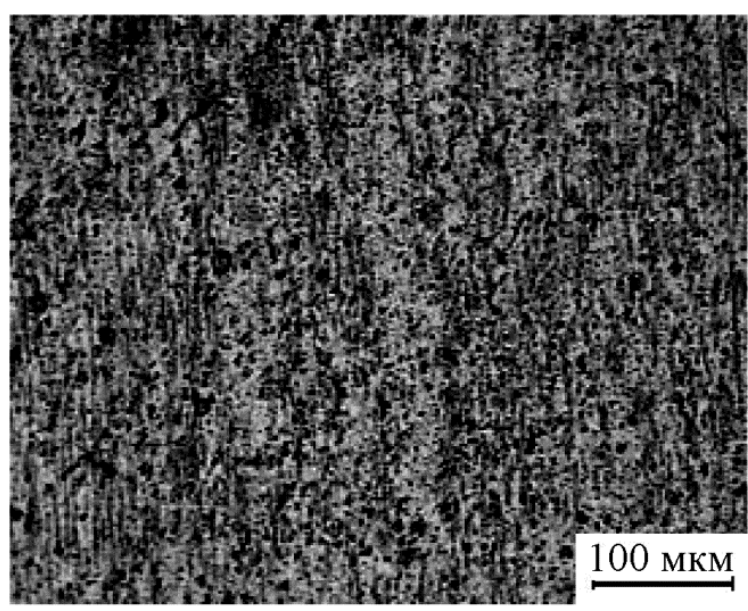

$\partial$

Рис. 2. Микроструктура после РКУПП: $a$ - после первого цикла деформирования; $\sigma, \boldsymbol{\theta}$ - после второго цикла; $2, \partial$ - после третьего цикла; в, $\partial-$ с применением промежуточных отжигов

Снижение временного сопротивления после 3 циклов РКУПП обусловлено наличием микротрещин, объединением их в глобальную трещину и последующим преждевременным разрушением. Как видно, образцы, подвергавшиеся промежуточному отжигу, и после второ-

Andreyachshenko V. A. Experience of applying shot-term intermediate anneals in equal-channel angular pressing of aluminum alloys // Diagnostics, Resource and Mechanics of materials and structures. - 2019. - Iss.1. - P. 43-54. DOI: 10.17804/2410-9908.2019.1.043-054. 
го и после третьего циклов деформирования, показывают более высокие показатели прочности при сохранении достаточного уровня относительного удлинения, по сравнению с образцами без промежуточных отжигов. Увеличение свойств вызвано частичной коагуляцией включений.

Анализ рельефа поверхностей разрушения образцов после растяжения показал, что на всех образцах после РКУПП без отжига присутствуют следы грубого волокнистого излома, поверхность разрушения расположена в двух и трех плоскостях. Поверхности разрушения образцов, обработанных с применением кратковременных отжигов, содержат сферические (коагулированные) частицы.

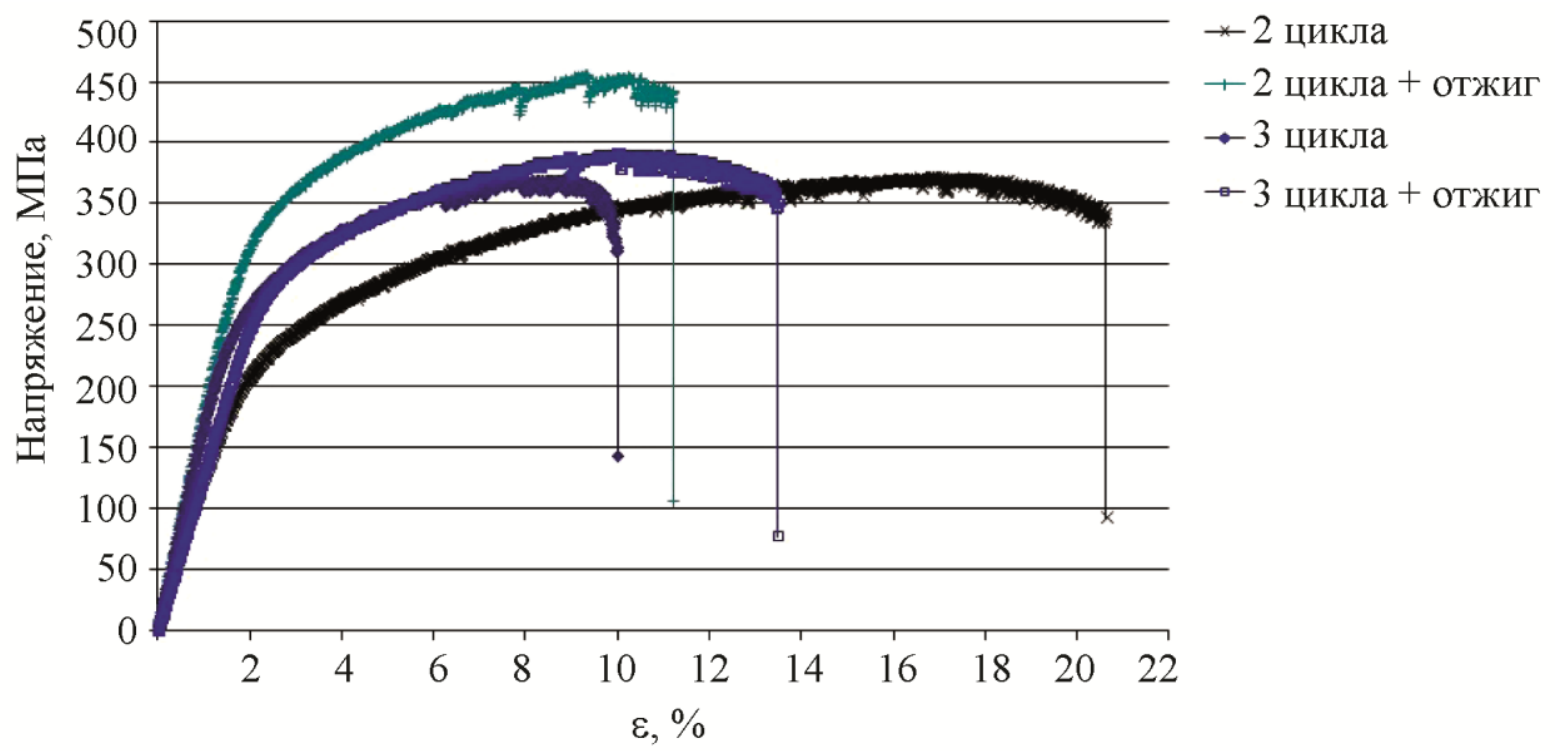

Рис. 3. Механические свойства сплава после РКУПП

При исследовании выявлено, что в результате реализации РКУПП происходит изменение значения пластичности. На рис. 5 приведены диаграммы зависимости $\Lambda_{\mathrm{p}}$ от степени деформации при РКУПП.

По данным, приведенным на рис. 5, видно, что кривые хорошо аппроксимируются полиномом второго порядка:

в области монотонных деформаций

$\mathrm{y}=-2,0814 \mathrm{x}^{2}+3,0636 \mathrm{x}-0,6499-\mathrm{R}^{2}=1-$ РКУПП;

$\mathrm{y}=-0,6237 \mathrm{x}^{2}+0,9276 \mathrm{x}-0,0467-\mathrm{R}^{2}=1-$ РКУПП + отжиг;

в области немонотонных деформаций

$\mathrm{y}=-0,8498 \mathrm{x}^{2}+1,1262 \mathrm{x}-0,0411-\mathrm{R}^{2}=1-$ для $\mathrm{T}=20^{\circ} \mathrm{C}$;

$\mathrm{y}=0,3549 \mathrm{x}^{2}-0,6568 \mathrm{x}+0,4642-\mathrm{R}^{2}=1-\mathrm{R}^{2}=1-$ РКУПП + отжиг.

Появление экстремума на кривых зависимости пластичности от степени деформации связано прежде всего со структурными изменениями, протекающими под влиянием РКУПП [24]. При увеличении степени деформации протекают два процесса, снижающие пластичность: упрочнение развитой дислокационной структурой и охрупчивание вторичными фазами, присутствующими в структуре металла. Существенной локализации деформации при испытании на растяжение не наблюдается. 


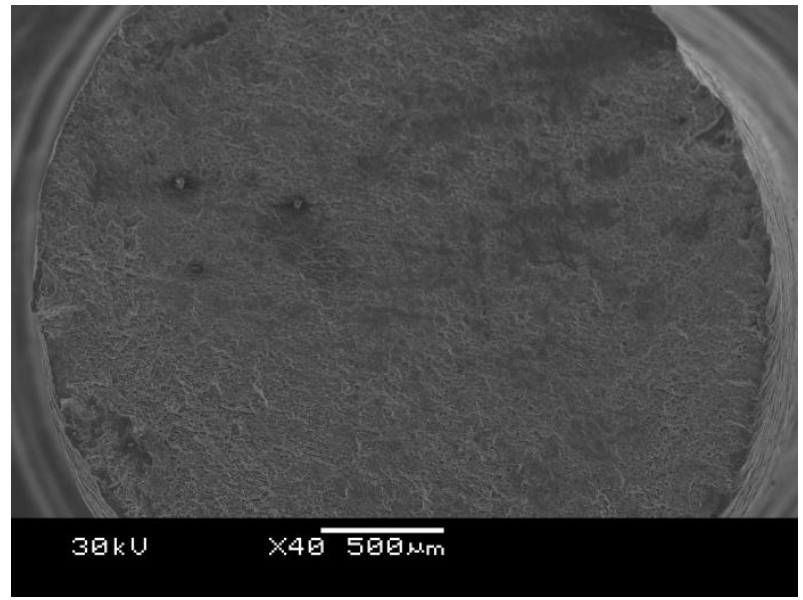

$a$

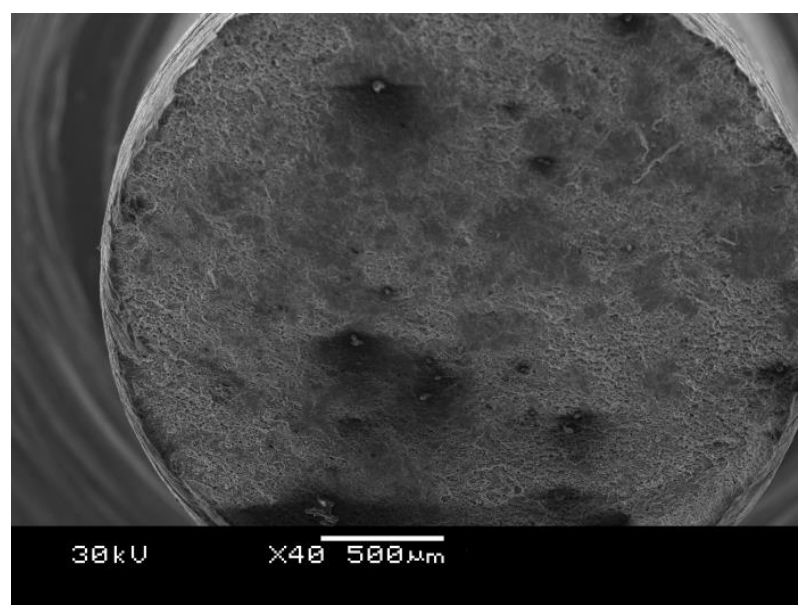

B

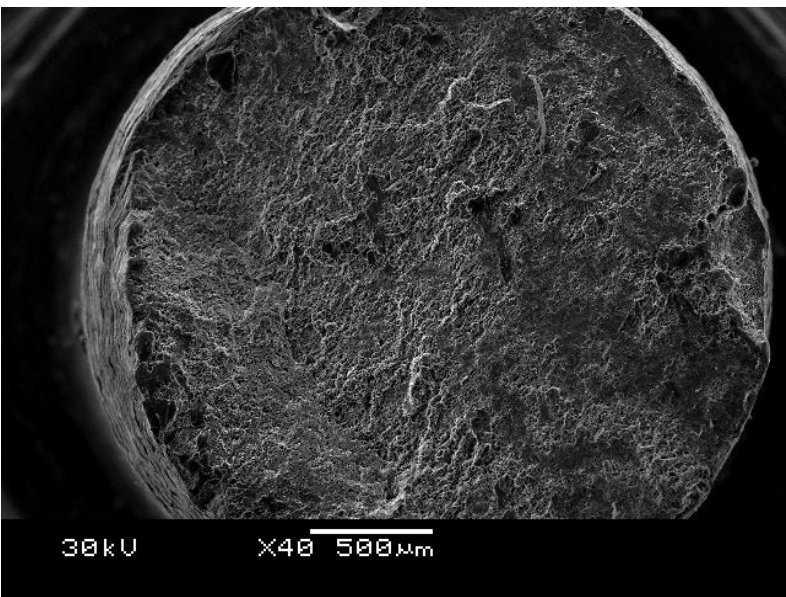

б

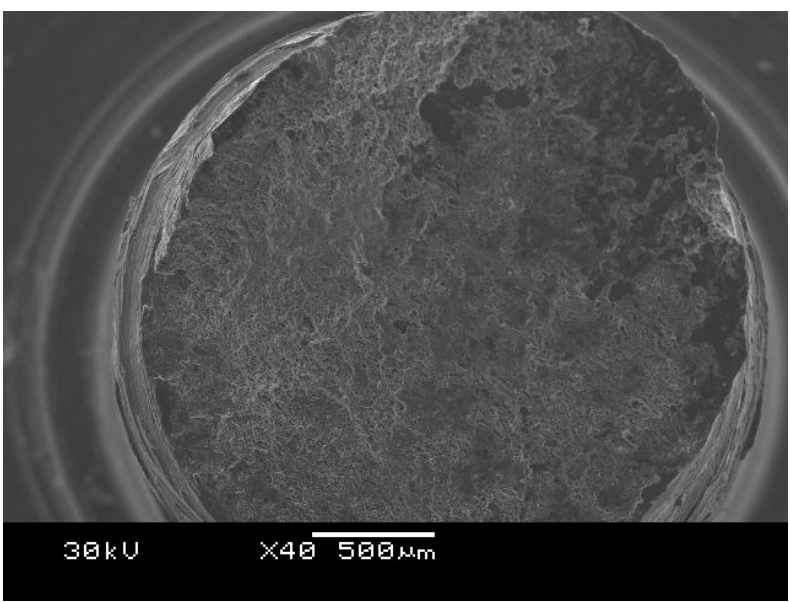
2

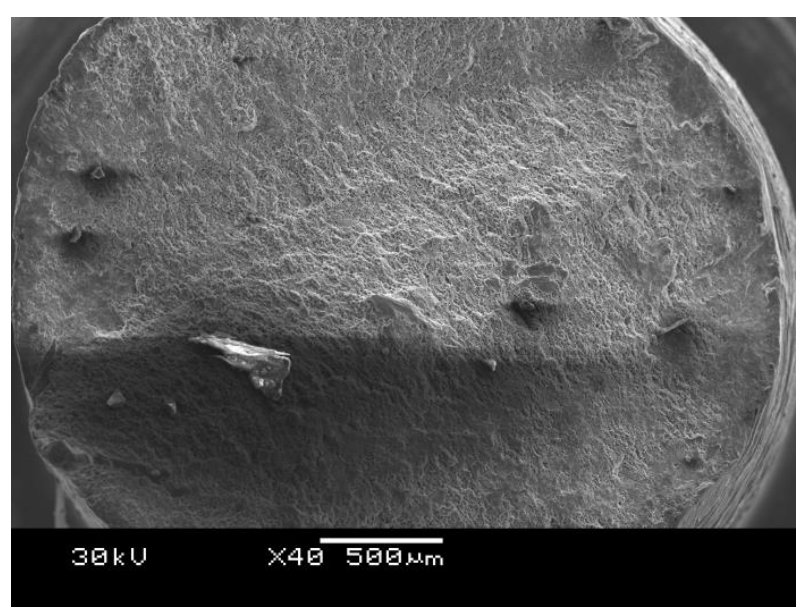

$\partial$

Рис. 4. Микрофрактография образцов после разрыва: $a$ - после первого цикла деформирования; $\sigma$, , - после второго цикла; г, $\partial$ - после третьего цикла; $b, \partial-\mathrm{c}$ применением промежуточных отжигов

Andreyachshenko V. A. Experience of applying shot-term intermediate anneals in equal-channel angular pressing of aluminum alloys // Diagnostics, Resource and Mechanics of materials and structures. - 2019. - Iss.1. - P. 43-54. DOI: $10.17804 / 2410-9908.2019 .1 .043-054$. 


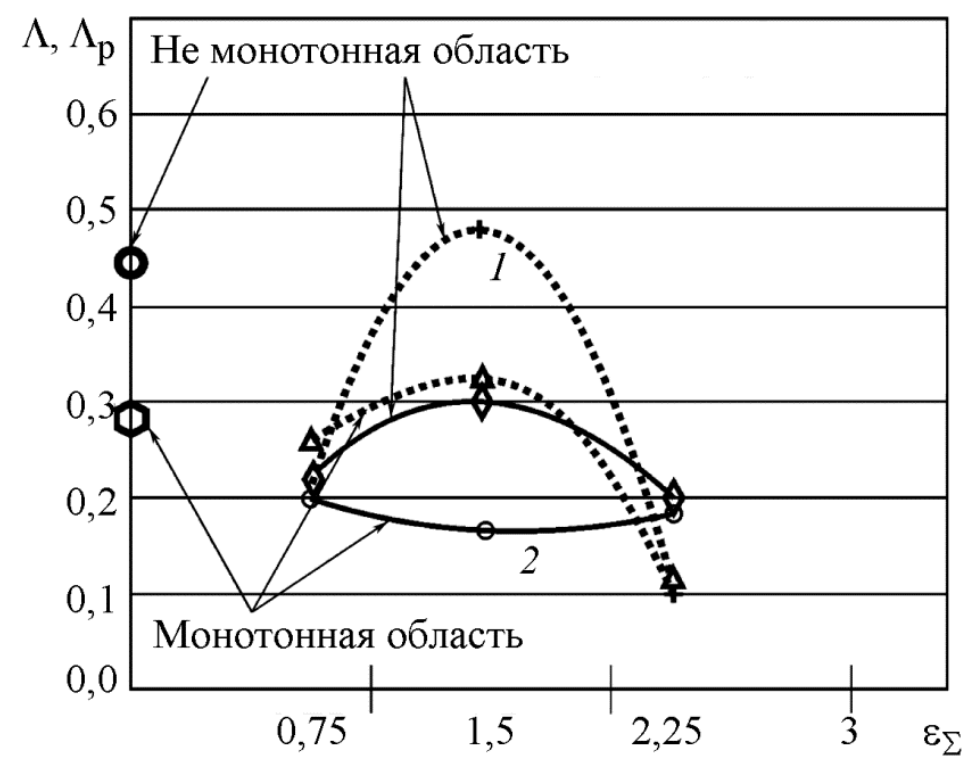

Рис. 5. Влияние суммарной степени деформации на пластичность:

$$
1 \text { - РКУПП; } 2 \text { - РКУПП + отжиг }
$$

В результате проведения двух циклов деформирования происходит формирование равномерной субультрамелкозернистой микроструктуры, что вызывает появление экстремума на кривых в состоянии после РКУПП и после деформирования с проведением дополнительных промежуточных отжигов. За счет повышения степени деформации происходит разрушение интерметаллидов и кремниевых частиц, зарождение микротрещин, что вызывает снижение пластичности.

В связи с тем, что при испытании на растяжение сплав не проявляет достаточную пластичность, также рассчитана величина $\Lambda$ в области монотонных деформаций, т.е. до образования шейки. Проведение первого промежуточного отжига вызывает падение пластичности в области монотонной деформации, образование шейки наступает достаточно рано. Благоприятное влияние дополнительных отжигов особо значимо после реализации 3 циклов РКУПП несмотря на провал пластичности после второго цикла РКУПП. Величина $\Lambda_{p}$ после обработки ниже исходного значения, что вызвано упрочнением в результате реализации РКУПП. Экстремумы на кривых пластичности в области монотонной деформации совпадают с экстремумами на кривых в области неоднородных деформаций (с образованием шейки).

\section{4. Выводы}

Показана целесообразность применения равноканального углового прессования для обработки доэвтектических сплавов. Проведение отжига способствует растворению упрочняющих интерметаллидных фаз и коагуляции кремниевых частиц. Высокая концентрация включений не позволяет выявить границы зерен $\alpha$-твердого раствора основной матрицы. При реализации РКУПП наблюдается рост прочностных свойств вплоть до 375 МПа, однако после трех циклов деформирования происходит резкое снижение относительного удлинения практически без увеличения прочности.

Проведенные исследования показали эффективность применения краткосрочных промежуточных отжигов при РКУПП при температуре $200{ }^{\circ} \mathrm{C}$ в течение 5 мин. В результате такой обработки дальнейший распад твердого раствора не успевает произойти, в связи с тем что твердый раствор не был пересыщен перед обработкой. Кратковременные отжиги способствуют коагуляции интерметаллидных включений, первичных и вторичных кремни- 
евых частиц. В результате обработки предел прочности после двух циклов РКУПП достигает 455 МПа при относительном остаточном удлинении $11 \%$. При проведении трех циклов деформирования наблюдается снижение прочности, но при этом значения остаются выше, чем у образцов, деформируемых без применения промежуточных отжигов. Предельная пластичность образцов с дополнительными отжигами, также превышает состояние после РКУПП без отжига, 0,2 и 0,12 соответственно.

В целом, с целью сохранения ресурса и улучшения доэвтектического силумина рекомендовано использование РКУПП с промежуточными отжигами с двумя циклами деформирования.

\section{Литература}

1. Новые высокопрочные алюминиевые сплавы и материалы / О. Г. Сенаторова, О. Е. Грушко, Е. А. Ткаченко, В. В. Антипов, И. И. Молостова, В. В. Сидельников, С. Ф. Легошина // Технология легких сплавов. - 2007. - № 2. - С. 17-24.

2. Миронов А. Е., Котова Е. Г. Разработка новых марок литейных алюминиевых антифрикционных сплавов для замены бронз в узлах трения // Известия Самарского научного центра Российской академии наук. - 2011. - Т. 13, № 4-3. - С. 1136-1140.

3. Освоение в промышленном производстве полуфабрикатов из перспективного алюминийлитиевого сплава В-1469 / Ю. Ю. Клочкова, О. Е. Грушко, Л. П. Ланцова, И. П. Бурляева, Б. В. Овсянников // Авиационные материалы и технологии. - 2011. - № 1 (18). - С. 8-12.

4. Доценко Ю. В., Селиверстов В. Ю. Особенности затвердевания отливок из алюминиевых сплавов при нарастающем давлении и модифицировании // Восточно-Европейский журнал передовых технологий. - 2012. - № 1 (5). - С. 18-22.

5. Оптимизация режимов искусственного старения кованых и катаных полуфабрикатов из жаропрочного алюминиевого сплава АК4-1Ч / А. А. Селиванов, К. В. Антипов, А. И. Асташкин, Б. В. Овсянников // Труды ВИАМ. - 2018. - № 4 (64). - С. 9-19. DOI: $10.18577 / 2307-6046-2018-0-4-9-19$.

6. Марукович Е. И., Стеценко В. Ю., Гутев А. П. Производство и применение силумина с глобулярным кремнием // Литье и металлургия. - 2017. - № 2 (87). - С. 15-19. DOI: $10.21122 / 1683-6065-2017-2-15-19$.

7. Прудников А. Н., Попова М. В., Прудников В. А. Воздействие деформации на структуру и свойства силуминов // Вестник Сибирского государственного индустриального университета. - 2017. - № 3 (21). - С. 11-17.

8. Богатов А. А., Нухов Д. Ш., Лещев И. В. Разработка инновационных процессов обработки металлов давлением на основе интенсивной знакопеременной деформации // Обработка сплошных и слоистых материалов. - 2016. - № 1 (44). - С. 35-43.

9. Микроструктура и механические свойства заэвтектического сплава $\mathrm{Al}-\mathrm{Si}$ после интенсивной пластической деформации / В. 3. Спусканюк, А. Л. Березина, В. И. Дубоделов, А. А. Давиденко, В. Н. Фикссен, К. И. Слива, Т. А. Монастырская, А. Н. Гангало // Металлофизика и новейшие технологии. - 2014. - Т. 36, № 5. - С. 649-660.

10. Бочвар Н. Р., Рохлин Л. Л., Тарытина И. Е. Совместное влияние интенсивной пластической деформации и холодной прокатки на механические свойства алюминиевых сплавов на основе системы Al-Mg 2 Si // Перспективные материалы. - 2018. - № 7. - С. 49-56. DOI: 10.30791/1028-978x-2018-7-49-56.

11. Автократова Е. В., Ситдиков О. Ш., Маркушев М. В. Сверхпластичность Al-Mg-Sc $(\mathrm{Zr})$ сплавов, подвергнутых интенсивной пластической деформации // Физика и механика материалов. - 2017. - Т. 33, № 1. - C. 19-28. - DOI: 10.18720/MPM.3312017_3.

12. Структура и свойства композита $\mathrm{A} 356-\mathrm{AlCuFe}$, полученного с использованием электромагнитного перемешивания / С. С. Полищук, А. Л. Березина, А. А. Давиденко, В. 3. Спус- 
канюк, В. Н. Фикссен, А. В. Ященко, В. В. Бурховецкий // Металлофизика и новейшие технологии. - 2014. - С. 1189-1205.

13. Resistance of submicrocrystalline aluminum alloys to high-rate deformation and fracture after dynamic channel angular pressing / I. G. Brodova, A. N. Petrova, S. V. Razorenov, E. V. Shorokhov // The Physics of Metals and Metallography. - 2015. - Vol. 116, iss. 5. P. 519-526. - DOI: 10.1134/S0031918X15050051.

14. Twist-channel angular pressing: effect of the strain path on grain refinement and mechanical properties of copper / R. Kocich, J. Fiala, I. Szurman, A. Macháčková, M. Mihola // Journal of Materials Science. - 2011. - Vol. 46, no. 24. - P. 7865-7876. - DOI: 10.1007/s10853-011-5768-1.

15. Способ деформационной обработки материалов и устройство для его осуществления: пат. 2146571 Рос. Федерация / Маркушев М. В., Слобода В. Н., Кайбышев О. А., Ин-т проблем сверхпластичности металлов РАН. - № 98107870/02 ; заявл. 17.04.1998 ; опубл. 20.03.00, Бюл. № 8 .

16. Mckenzie P. W. J., Lapovok R. ECAP with back pressure for optimum strength and ductility in aluminium alloy 6016. Part 2: Mechanical properties and texture // Acta Materialia. - 2010. Vol. 58 (9). - P. 3212-3222. - DOI: 10.1016/j.actamat.2010.01.037.

17. Optimisation of interface formation by shear inclination: Example of aluminium-copper hybrid produced by ECAP with back-pressure / Alexander E. Medvedev, Rimma Lapovok, Eric Koch, Heinz Werner Höppel, Mathias Göken // Materials \& Design. - 2018. - Vol. 146. P. 142-151. - DOI: 10.1016/j.matdes.2018.03.021.

18. Naizabekov A. B., Andreyashchenko V. A. Evaluation of possibility for increase of mechanical characteristics of the $\mathrm{Al}-\mathrm{Fe}-\mathrm{Si}-\mathrm{Mn}$ alloy by equal-channel angular pressing // Metallurgist. 2013. - Vol. 57, no. 1-2. - P. 159-163. - DOI: 10.1007/s11015-013-9706-0.

19. Influence of the supersaturated silicon solid solution concentration on the effectiveness of severe plastic deformation processing in Al-7 wt. \% Si casting alloy / C. M. Cepeda-Jiménez, J. M. García-Infanta, A. P. Zhilyaev, O. A. Ruano, Fernando Carreño, Alexander Zhilyaev, Oscar Ruano // Materials Science and Engineering: A. - 2011. - Vol. 528, no. 27. - P. 7938-7947. DOI: 10.1016/j.msea.2011.07.016.

20. Пластометрические исследования металлов / Н. А. Мочалов, А. М. Галкин, С. Н. Мочалов, Д. Ю. Парфенов. - М. : Интермет Инжиниринг, 2003. - 318 с.

21. Assessment of homogeneity of the shear-strain pattern in Al-7wt \% Si casting alloy processed by high-pressure torsion / C. M. Cepeda-Jimenez, A. Orozco-Caballero, J. M. GarciaInfanta, A. P. Zhilyaev, O. A. Ruano, F. Carreno // Materials Science and Engineering A, 2014. Vol. 597. - P. 102-110. - DOI: 10.1016/j.msea.2013.12.072.

22. Andreyachshenko V., Naizabekov A. The technology of equal channel angle backpressure extrusion for deformation iron and aluminium alloys // Proceedings Book of 3rd International Conference NANOCON. - Czech Republic, Tanger Ltd., 2011. - P. 246-252.

23. Naizabekov A., Andreyachshenko V., Kliber J. Forming of microstructure of the Al-Si-Fe-Mn system alloy by equal channel angular pressing with backpressure // 21st International Conference on Metallurgy and Materials Metal-2012, Brno Czech Republic : proceedings, 2012. - P. 391-395.

24. Найзабеков А. Б., Андреященко В. А. Микроструктурные и механические характеристики сплава Al-Fe-Si-Mn подвергнутого равноканальному угловому прессованию // X Международная научная конференция «Перспективные технологии, оборудование и аналитические системы для материаловедения и наноматериалов» : сб. трудов. - Алматы, Республика Казахстан, 2013. - С. 114-122. 